\title{
Notas y nuevos sitios de encuentro de Caluromys derbianus (Marsupialia: Didelphidae) en Honduras
}

\author{
Notes and noteworthy records of Caluromys derbianus \\ (Marsupialia: Didelphidae) in Honduras
}

\author{
Leonel E. Marineros ${ }^{1}$, Hermes L. Vega ${ }^{2}$, James Adams³ ${ }^{3}$ Mayron McKewy Mejía ${ }^{4}$
}

\section{Resumen}

\begin{abstract}
Introducción: Caluromys derbianus (Waterhouse, 1841) es uno de los marsupiales presentes en Honduras documentado desde 1942. Sin embargo, son escasos los registros en el país. Objetivo: Actualizar el mapa de distribución de este marsupial en Honduras, brindar información sobre nombres locales, así como datos de depredación y reproducción documentados dentro de Honduras. Metodología: Documentar la presencia de este animal a través de los encuentros que los autores y colaboradores han tenido en Honduras en diferentes labores de monitoreo, inventarios, limpieza y destronque de servidumbres. Al final, cada animal fue georreferenciado, fotografiado y liberado en el caso de encuentros con animales vivos. Resultados: Se lograron obtener 29 nuevas locaciones de encuentros, todos asociados con bosques latifoliados por debajo de los 1,082 metros de elevación. La mayoría de los encuentros se dieron en agropaisajes con altos niveles de bosques fragmentados. Se discuten aspectos de distribución, nombres locales y conservación en el país. Se presenta un mapa de distribución con los sitios históricos y nuevos. Se incorporan los departamentos de Copán, El Paraíso y Yoro con nuevos records departamentales. Se documentaron tres casos de hembras con crías, siendo el máximo de tres lactantes. Finalmente se informa un caso de depredación por parte del búho Pulsatrix perspecillata.
\end{abstract}

Palabras clave: Central American woolly oposum, Depredación, Pulsatrix perspicillata, Records departamentales, Reproducción, Zorra lanosa centroamericana.

\begin{abstract}
Introduction: The Central American woolly opossum Caluromys derbianus (Waterhouse, 1841) is one of the marsupials of Honduras documented since 1942. However, there are few records in the country. Objective: The objective of this study was to create an updated distribution map of this marsupial in Honduras, provide information on local names, and document reproduction and predation events. Methodology: Authors and collaborators documented encounters of $C$. derbianus in Honduras while working on monitoring, inventorying, or clearing vegetation on easements. In the case of live encounters, each animal was georeferenced, photographed, and released. Results: We encountered 28 new locations for $C$. derbianus, all associated with broadleaf forests at less than 1,090 meters above sea level. Most encounters occurred in agricultural areas with high levels of fragmented forests. We discuss aspects of distribution, local names, and conservation in the country. We created a distribution map with historical and new sites (the departments of Copan, El Paraíso and Yoro with new departmental records are incorporated). Three cases of females with young were documented, with the maximum of three offspring. Finally, we documented one case of predation by a Spectacled Owl (Pulsatrix perspecillata).
\end{abstract}

Keywords: Departmental records, Reproduction, Predation, Pulsatrix perspicillata, Woolly opossum.

Colaborador del Instituto INCEBIO, Tegucigalpa, Honduras. e-mail: lmarineros@gmail.com

Mancomunidad Parque Nacional Celaque (MAPANCE), ciudad de Gracias, Lempira, Honduras.

Hotel Pico Bonito Lodge, La Ceiba, Atlántida, Honduras.

Comité Científico de la Asociación Hondureña de Ornitología (ASHO), Tegucigalpa, Honduras.

Fecha recepción: Julio 21, $2015 \quad$ Fecha aprobación: Diciembre 6, $2015 \quad$ Editor Asociado: Mantilla-Meluk H.

(c) Rev. Biodivers. Neotrop. 2016; 6 (1): 77-84 


\section{Introducción}

Los marsupiales del género Caluromys Allen 1900 son parte de un linaje de la familia Didelphidae propios del nuevo mundo (Voss y Jansa 2009). En este género se incluyen tres especies, Caluromys derbianus Waterhouse 1841, C. lanatus Olfers 1818 y C. philander Linnaeus 1758, estas especies están ampliamente distribuidos en Centro y Suramérica (Gardner 2008).La zarigüeya lanosa centroamericana $C$. derbianus Waterhouse 1841, tiene un rango de distribución desde Veracruz, México, toda Centroamérica hasta el Oeste de Ecuador y el Valle del Cauca en Colombia (Hall y Kelson 1959, Bucher y Hoffman 1980, Reid 2009). Se le ha encontrado desde las planicies costeras hasta elevaciones que alcanzan los 2600 metros de elevación (Eisemberg 1989; Voss y Jansa 2009). Se reconocen 7 subespecies en toda su área de distribución en el Neotrópico, dos de ellas en Honduras $C$. $d$. canutus, en la Mosquitia hondureña, y $C . d$. fervidus en el resto del país. Una tercera subespecie $C$. $d$. pallidus puede aparecer a lo largo de la frontera con Nicaragua (Hall y Kelson 1959, Bucher y Hoffman 1980).

Estas subespecies han sido descritas con base en características morfológicas externas tales como cuerpo, cara, dorso, cola y coloración de las patas. Estas características usualmente presentan variación geográfica dentro de la especie. Las subespecies de Caluromys derbianus fueron descritas basadas en esas características morfológicas comparando especímenes de diferentes partes dentro de su rango de distribución y comparadas con el holotipo (Fonseca y Astúa 2015).

Este marsupial didélfido ha sido poco registrado en Honduras. Los primeros registros los presentó Goodwin en 1942 a través de 3 especímenes de museo. Uno de ellos proveniente de Ilama en Santa Bárbara, otro de Chamelecón en Cortés y otro de Catacamas en Olancho. Los últimos registros publicados de este animal en Honduras fueron dados por Marineros y Martínez (1998) a través de nueve avistamientos sin colecta de especímenes. Este marsupial habita en bosques deciduos y húmedos, tanto de tierras bajas como bosques húmedos de altura. Es predominantemente arborícola, nocturno y solitario, encontrándose en bosques maduros, así como también, en bosques perturbados. Posee hábitos omní- voros y en la dieta incorpora frutas, hojas, semillas, insectos, otros invertebrados y posiblemente carroña (Bucher y Hoffmann 1980).

El objetivo de este estudio fue actualizar el mapa de distribución de este marsupial en Honduras reuniendo registros de la literatura con nuevos avistamientos, así como brindar información sobre nombres locales usados en el país, proporcionar datos de depredación y reproducción documentados dentro de Honduras.

\section{Metodología}

La metodología consistió en tres etapas:

1. A través de visitas a diferentes partes de Honduras, donde se realizaron entrevistas a personal técnico de campo de diferentes proyectos, quienes aportaron material fotográfico como evidencia, tanto de ejemplares encontrados vivos como muertos, se identificó la especie con base en sus características morfológicas conspicuas, que la distingue y diferencia de las otras zarigüeyas hondureñas. La verificación de la especie fue validada con base en las características corporales propuestas por Bucher y Hoffmann (1980), Eisemberg (1989) y por Reid (2009) quienes lo describen como un marsupial mediano (HB: 247-321 mm, T: 271-457 mm), con pelaje denso y lanoso de color café claro, siendo el pelaje blanco en las extremidades, costado y vientre. Los ojos de color café con pupilas negras. Las patas, nariz y orejas presentan una tonalidad rosada. La cola es prensil y constituye el $58 \%$ a $67 \%$ de la longitud del animal. La cola presenta pelaje lanoso, pero la mayor parte es desnuda con coloración blancuzca en su parte distal y con manchas negras después de la parte lanosa. En la frente se presenta una línea oscura.

2. Aprovechando diferentes labores de campo, que incluyeron monitoreo biológico, raleo de árboles e inventarios faunísticos, los autores documentaron y registraron fotográficamente la presencia de $C$. derbianus en varios departamentos de Honduras. Según el tipo de contacto, los encuentros fueron registrados como avistamiento directo, capturas con trampa, animales atropellados y carcasas. Se documentó el tipo de actividad donde el observador encontró el animal, se georreferenció el sitio, procedió a fotografiarlo y a liberarlo si así fuera el caso. 
3. Simultáneamente, se realizaron entrevistas informales no estructuradas con residentes de 41 sitios del país. La entrevista fue orientada a encontrar usos, perjuicios y nombres locales de la especie. Inicialmente con base en fotos o dibujos de la zarigüeya lanosa se indagó entre algunos miembros de la población (principalmente hombres adultos residentes), sobre la presencia de este marsupial. Una respuesta positiva permitió conversar sobre su nombre local, uso, frecuencia y perjuicios posibles.

\section{Resultados}

Sitios históricos. Se encontraron 11 sitios históricos para ocho departamentos de Honduras (Tabla 1). Los registros más antiguos son los de Goodwin en su estudio de 1942, quien documenta dos sitios ubicados en el departamento de Santa Bárbara y uno en el departamento de Olancho, basados en pieles de museo en Estados Unidos (AMNH 126134, USNM 148749, AMNH 126980).

Marineros y Martínez (1998) mencionan ocho sitios en seis departamentos, Atlántida, Comayagua, Gracias a Dios, Olancho, Francisco Morazán y Cortés. Los datos presentados en este documento, fueron dados por información de avistamientos de campo sin capturas de especímenes. En este documento, Marineros y Martínez (1998) también mencionan a un animal encontrado cautivo en una feria colegial en Santa Rosa de Copán. El espécimen registrado en Comayagua se trató de un espécimen de museo en la colección de la Universidad Nacional Autónoma de Honduras (UNAH), preservado en formalina y sin datos de origen más que la palabra Comayagua.

Nuevos sitios. La Tabla 2 detalla la ubicación de 29 nuevos encuentros de $C$. derbianus por departamento, junto con los datos de nombre del técnico que lo reporta, municipio y el tipo de contacto, avistamiento directo, registro de cuero de animal, encuentro de animal atropellado o carcasa o bien de material fotográfico como evidencia. Se registra una ubicación en UTM y la elevación del sitio. En este caso, se incorporan Copán, Yoro y El Paraíso como nuevos departamentos en la distribución de este marsupial; todos los registros están debajo de los 1090 de elevación.

Nombres locales. No hay un nombre vernáculo para este marsupial en Honduras. La mayoría de los entrevistados no dieron un nombre local para este didélfido. En entrevistas realizadas, le dieron el nombre de otros mamíferos hondureños. Para el caso en la Reserva Biológica El Chile, departamento de Francisco Morazán, un grupo de personas lo identificó con el nombre "ratón guazalo", ese nombre también lo dan a las especies del género Marmosa. En las montañas cercanas a Lancetilla, municipio de Tela, le llamaron "pereza", nombre usado también en Hon-

Tabla 1. Sitios históricos para Caluromys derbianus en Honduras

\begin{tabular}{|c|c|c|c|c|c|}
\hline & Departamento & Sitio & Municipio & Reporte & Referencia \\
\hline 1 & Atlántida & $\begin{array}{l}\text { Col. Las Acacias a orillas } \\
\text { del río Cangrejal }\end{array}$ & La Ceiba & $\begin{array}{l}\text { Iván Rivera y } \\
\text { L. Marineros }\end{array}$ & $\begin{array}{l}\text { Marineros y } \\
\text { Martínez } 1998\end{array}$ \\
\hline 2 & Comayagua & Sin datos & Sin datos & Museo UNAH & $\begin{array}{l}\text { Marineros y } \\
\text { Martínez } 1998\end{array}$ \\
\hline 3 & Copán & $\begin{array}{l}\text { Feria de colegio en Santa } \\
\text { Rosa }\end{array}$ & Santa Rosa de Copán & $\begin{array}{l}\text { L. Marineros, } \\
\text { Sergio Midence }\end{array}$ & $\begin{array}{l}\text { Marineros y } \\
\text { Martínez } 1998\end{array}$ \\
\hline 4 & Cortés & Chamelecón & SPS & USNM 148749 & Goodwin 1942 \\
\hline 5 & Cortés & Santa Cruz de Yojoa & Santa Cruz de Yojoa & Efraín Villeda & $\begin{array}{l}\text { Marineros y } \\
\text { Martínez } 1998\end{array}$ \\
\hline 6 & $\begin{array}{l}\text { Francisco } \\
\text { Morazán }\end{array}$ & Reserva El Chile & Guaimaca & L. Marineros & $\begin{array}{l}\text { Marineros y } \\
\text { Martínez } 1998\end{array}$ \\
\hline 7 & Gracias a Dios & Puerto Lempira & Puerto Lempira & Becky Myton & $\begin{array}{l}\text { Marineros y } \\
\text { Martínez } 1998\end{array}$ \\
\hline 8 & Olancho & Curso medio del Patuca & Catacamas & $\begin{array}{l}\text { Napoleón } \\
\text { Morazán }\end{array}$ & $\begin{array}{l}\text { Marineros y } \\
\text { Martínez } 1998\end{array}$ \\
\hline 9 & Olancho & Agalta & Gualaco & D. Anderson & $\begin{array}{l}\text { Marineros y } \\
\text { Martínez } 1998\end{array}$ \\
\hline 10 & Olancho & Catacamas & Catacamas & AMNH 126980 & Goodwin 1942 \\
\hline 11 & Santa Bárbara & Ilama & Ilama & AMNH 126134 & Goodwin 1942 \\
\hline
\end{tabular}


Tabla 2. Nuevos registros de encuentros de Caluromys derbianus en Honduras hasta 2015

\begin{tabular}{|c|c|c|c|c|c|c|}
\hline \multicolumn{2}{|c|}{ Departamento } & \multirow[t]{2}{*}{ Sitio } & \multirow{2}{*}{$\begin{array}{l}\text { Municipio } \\
\text { El Porvenir }\end{array}$} & \multirow{2}{*}{$\begin{array}{c}\text { Reporte } \\
\text { Foto de Danny Ordoñez }\end{array}$} & \multirow{2}{*}{$\begin{array}{c}\begin{array}{c}\text { Coordenadas } \\
\text { en UTM }\end{array} \\
16 P 509510-1741745\end{array}$} & \multirow{2}{*}{$\begin{array}{c}\begin{array}{c}\text { Elevación } \\
\text { msnm }\end{array} \\
4\end{array}$} \\
\hline 1 & Atlántida & & & & & \\
\hline 2 & Atlántida & $\begin{array}{l}\text { Parque Nacional Nombre de } \\
\text { Dios, al sur de la comunidad } \\
\text { de Roma }\end{array}$ & La Ceiba & $\begin{array}{l}\text { Avistamiento de Josué Ramos } \\
\text { Galdámez }\end{array}$ & $16 \mathrm{P} 542651-1744465$ & 30 \\
\hline 3 & Atlántida & Río Santiago & San Francisco & Foto de James Adams & 16P 493108-1723123 & 82 \\
\hline 4 & Atlántida & $\begin{array}{l}\text { Plantaciones de caoba, cerca } \\
\text { de la entrada Al Jardín Botá- } \\
\text { nico de Lancetilla }\end{array}$ & & Foto de Ciro Vladimir Navarro & 16P 451336-1742185 & 25 \\
\hline 5 & Atlántida & Reserva de Lancetilla & Tela & Foto de Wendy Cerrato & 16P 451423-1738964 & 86 \\
\hline 6 & Atlántida & Aldea Perú & Tela & Foto de Jennifer Martínez & 16P 529990-1744355 & 44 \\
\hline 7 & Comayagua & $\begin{array}{l}\text { Aldea Monte Redondo, carre- } \\
\text { tera a Lajas }\end{array}$ & $\begin{array}{l}\text { La Ceiba } \\
\text { La Libertad }\end{array}$ & Foto de Roberto Downing & 16P 427911-1639549 & 324 \\
\hline 8 & Copán & $\begin{array}{l}\text { Parque Arqueológico Ruinas } \\
\text { de Copán }\end{array}$ & Copán Ruinas & Animal atropellado L. Marineros & $16 \mathrm{P} 2$ & 605 \\
\hline 9 & Copán & $\begin{array}{l}\text { Cerca de la Estela } 13 \text { de } \\
\text { Copán }\end{array}$ & Santa Rita & Animal atropellado L. Marineros & 16P 273121-1644437 & 651 \\
\hline 10 & Copán & Sitio Arqueológico Rastrojón & Copán Ruinas & $\begin{array}{l}\text { Avistamiento de Jonathan } \\
\text { Hernández, Hermes Vega, Héctor } \\
\text { Portillo, Fausto Elvir, L. Marineros }\end{array}$ & 16P 270826-1643660 & 647 \\
\hline 11 & Cortés & Santa Cruz de Yojoa & Santa Cruz & Mario Espinal, Banny Quezada & $16 \mathrm{P} 402542-1658420$ & 382 \\
\hline 12 & Cortés & Carretera a Pulhapanzak & $\begin{array}{l}\text { San Francisco } \\
\text { de Yojoa }\end{array}$ & $\begin{array}{l}\text { Animal muerto en carretera, foto de } \\
\text { Roberto Downing }\end{array}$ & 16P 392128- 1660629 & 211 \\
\hline 13 & Cortés & Colonia Campisa & San Pedro Sula & $\begin{array}{l}\text { Animal atropellado Pablo } \\
\text { Bedrossian }\end{array}$ & $16 \mathrm{P} 393686-1718166$ & 111 \\
\hline 14 & Cortés & Cerro Campisa & San Pedro Sula & José Fernández y Pablo Bedrossian & 16P 393203-1717620 & 35 \\
\hline 15 & Cortés & Aldea La Fe & Peña Blanca & $\begin{array}{l}\text { Una hembra con crías. Foto de } \\
\text { Saby Cruz }\end{array}$ & 16P 386879-1657346 & 741 \\
\hline 16 & Cortés & El Paraíso, valle de Cuyamel & Omoa & Roberto Downing & 16P $363237-1725318$ & 304 \\
\hline 17 & El Paraíso & Cerro de Apagüiz & Danlí & Mayron Mejía & $8-1550302$ & 984 \\
\hline 18 & Olancho & Talgua & Catacamas & Cuero, Olvin Calixto Ordóñez & 16P 621442-1646733 & 540 \\
\hline 19 & Olancho & $\begin{array}{l}\text { Cerca de Gualaco, animal en } \\
\text { venta en la calle }\end{array}$ & Gualaco & Avistamiento de Robert Gallardo & 16P 600624-6600629 & 632 \\
\hline 20 & Olancho & $\begin{array}{l}\text { UNA-G Escuela Nacional de } \\
\text { Agricultura y Ganadería }\end{array}$ & Catacamas & $\begin{array}{l}\text { Animal vivo encontrado al caer } \\
\text { una rama de guanacaste. Foto de } \\
\text { Bayardo Alemán }\end{array}$ & 16P 624415-1639484 & 350 \\
\hline 21 & Olancho & Laguna de Huyaste & Catacamas & $\begin{array}{l}\text { Animal encontrado muerto. Foto de } \\
\text { Juan Pablo Suazo }\end{array}$ & 16P 635808-1637883 & 357 \\
\hline 22 & Santa Bárbara & Quebrada Onda & San L & Foto de Carlos O'Reilly & $16 \mathrm{P} 34 \varepsilon$ & 420 \\
\hline 23 & Santa Bárbara & Valle de Tencóa & Santa Bárbara & $\begin{array}{l}\text { Atropellado. Foto de Banny } \\
\text { Quezada }\end{array}$ & 16P 365912-1642945 & 180 \\
\hline 24 & Santa Bárbara & Aldea Tierra Blanca & Nueva Celilac & $\begin{array}{l}\text { Avistamiento nocturno. Hermes } \\
\text { Vega }\end{array}$ & 16P 356593-1653815 & 460 \\
\hline 25 & Santa Bárbara & $\begin{array}{l}\text { Quebrada La Chácara, rumbo } \\
\text { a cerro Montuca, San Nicolás, } \\
\text { Santa Bárbara }\end{array}$ & San Nicolás & $\begin{array}{l}\text { Hembra con crías encontrada al } \\
\text { podar árboles. Foto de Hermes } \\
\text { Vega }\end{array}$ & 16P 357116-1651440 & 534 \\
\hline 26 & Santa Bárbara & Aldea Loma Alta & Protección & Bertín Lazo & 16P 321914- 1663310 & 1090 \\
\hline 27 & Santa Bárbara & $\begin{array}{l}\text { La Vegona, Rio Chamelecón. } \\
\text { Al sureste de Sula }\end{array}$ & Macuelizo & Avistamiento de Roberto Downing & $16 \mathrm{P} 334481-1683908$ & 250 \\
\hline 28 & Santa Bárbara & $\begin{array}{l}\text { San Ramón, orilla del río } \\
\text { Gualcarque }\end{array}$ & $\begin{array}{l}\text { San Francisco } \\
\text { de Ojuera }\end{array}$ & Avistamiento de Roberto Downing & 16P 371027-1622188 & 560 \\
\hline 29 & Yoro & $\begin{array}{l}\text { Loma Larga, amortiguamiento } \\
\text { de RVS Texiguat }\end{array}$ & Morazán & Foto de Rómulo Gutiérrez & 16P $452410-1711879$ & 586 \\
\hline
\end{tabular}

duras para los osos perezosos (familias Bradypodidae y Megalonychidae). También en esta región caribeña, lo identificaron con el nombre de "comadreja", nombre que también es utilizado para el mustélido Mustela frenata. En los departamentos de Copán y Santa Bárbara, varios le llamaron "guazalo de agua" o "perro de agua" (un nombre no apropiado para esta especie arborícola) y que también lo usan en esta zona para Chironectes minimus. En la zona Tawahka de la Mosquitia hondureña se les da el nombre de "suksuk" a este marsupial y a otros animales nocturnos (comunicación personal de Virginia Maklim Salinas, indígena Tawahka).

Depredación. Pocos textos brindan información sobre depredación a la que es sujeta la especie $C$. derbianus. Bianchi et at. (2011) confirma la depredación de esta especie por ocelotes (Leopardus pardalis). Otras especies de zarigüeyas lanosas suramericanas de este género también son depredadas por felinos silvestres, como Leopardus wiedii, Puma yagouaroundi, P. cocolor y Panthera onca (Moreno et al. 2006).

En Honduras, Joel Mejía compartió una foto 
tomada en las inmediaciones del hotel Pico Bonito Lodge (Parque Nacional Pico Bonito, cordillera de Nombre de Dios) en el Caribe de Honduras, donde se aprecia a un búho Pulsatrix perspicillata depredando a un $C$. derbianus en plena mañana (Figura 1). En la revisión de la dieta confirmada de esta rapaz nocturna, se han documentado presas grandes y medianas incluyendo zarigüeyas (Gómez de Silva et al. 1997); sin embargo, este es el primer registro documentado de depredación de este búho sobre $C$. derbianus.

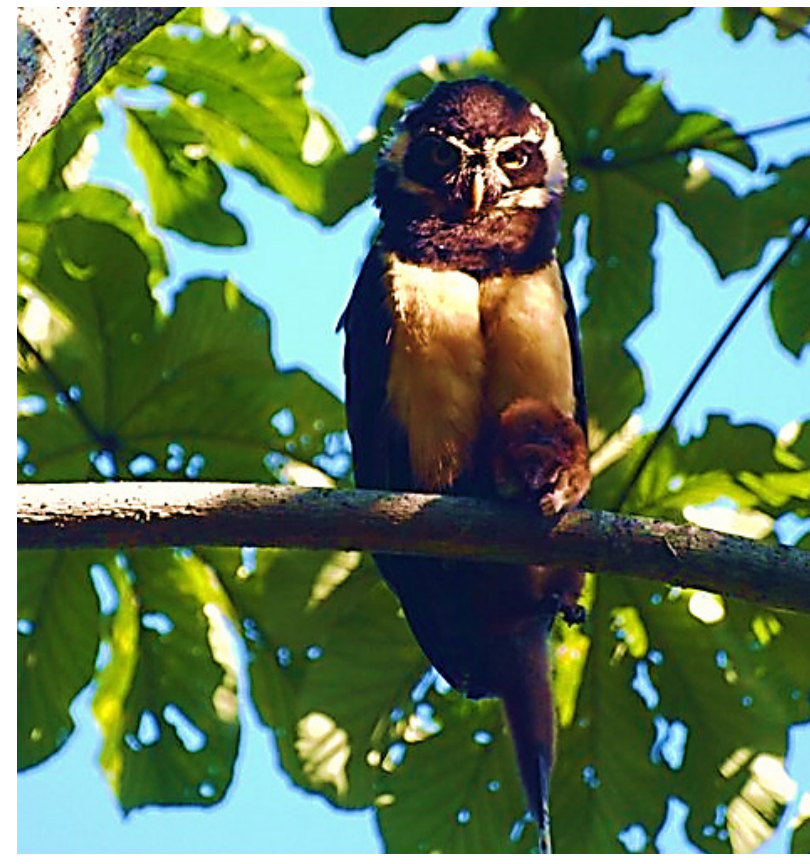

Figura 1. Un búho Pulsatrix perspicillata depredando a un Caluromys derbianus en el Parque Nacional Pico Bonito. Foto de Joel Mejía.

Datos reproductivos. Comparado con otros marsupiales, $C$. derbianus no es muy prolífico. Collins (1973) comentó sobre partos de tres crías. Philips y Jones (1968) dicen que esta zarigüeya se reproduce a lo largo del año en Nicaragua. En este estudio, se obtuvieron datos de presencia de crías desde mayo hasta agosto en Honduras. Se lograron documentar 3 subjuveniles lactantes con su madre en el mes de junio del 2013 en Peña Blanca, Cortés, documentado por Saby Cruz (Figura 2). Por su parte, Hermes Vega fotografió otra hembra con una sola cría lactante en el mes de mayo del 2014 en Tierra Blanca (Santa Bárbara) y Mayron Mckewy Mejía fotografió una hembra con dos crías en el mes de agosto del 2014 en Piedra de Apagüiz, departamento de El Paraíso. Bayardo Alemán, nos envió una foto de Catacamas

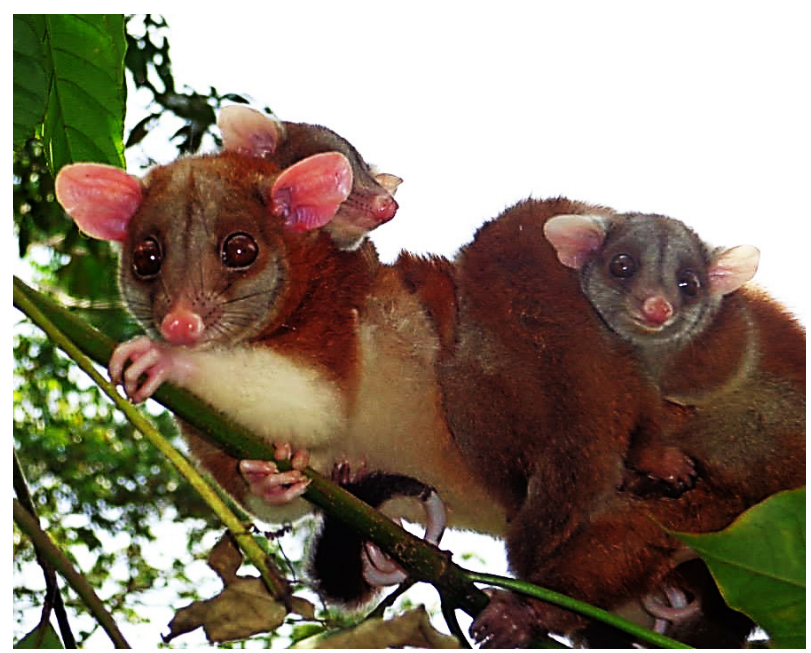

Figura 2. Madre y tres crías encontradas en Aldea La Fe, municipio de Peña Blanca, Cortés, en junio de 2013. Foto de Saby Cruz.

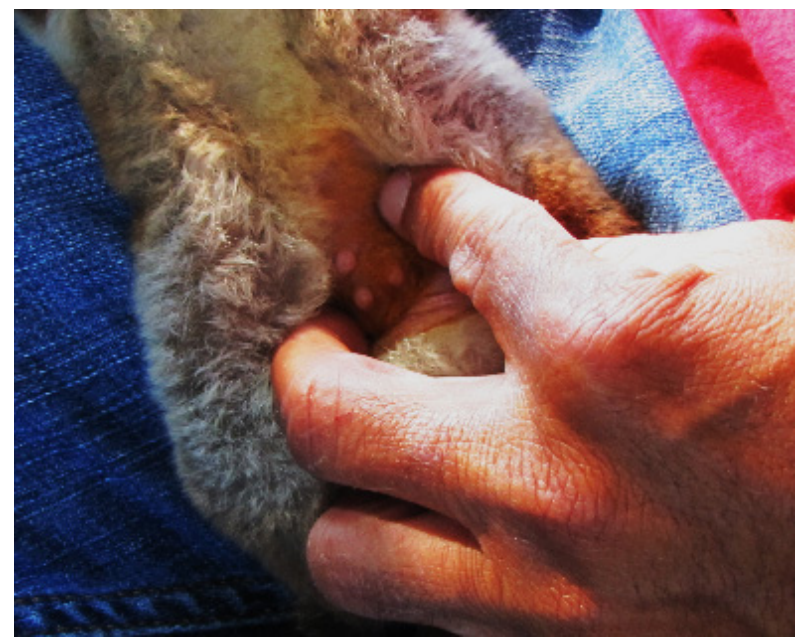

Figura 3. Hembra no lactante de $C$. derbianus.

Foto tomada en UNA-G, Catacamas por Bayardo Alemán.

de una hembra mostrando los tres pezones ventrales no lactantes (Figura 3).

Refugio. Estos marsupiales son arborícolas estrictamente nocturnos (Eisemberg 1989). Las madrigueras documentadas en Honduras fueron encontradas en huecos de árboles entre 3 y 5 metros de altura. Los ejemplares procedentes de Peña Blanca, Tierra Blanca y Piedra de Apagüiz, fueron documentados porque destruyeron sus madrigueras debido a que estaban realizando actividades de mantenimiento y poda de árboles. En el caso del ejemplar de Apagüiz, la madre se encontraba dormida atrás de un racimo de bananos dentro de un cafetal de sombra. El ejemplar de la Figura 4, tomada en el Parque Nacional Pico Bonito, 


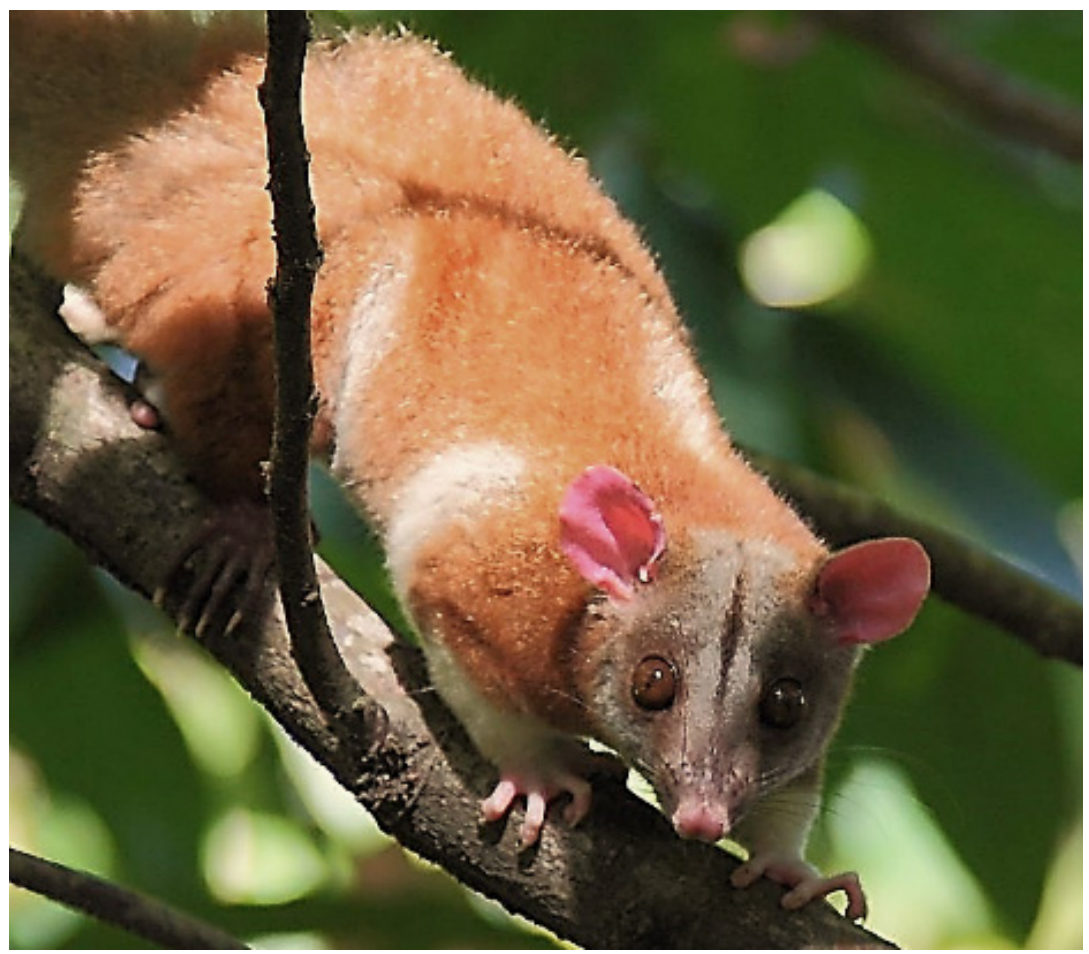

Figura 4. Caluromys derbianus tomado en Río Santiago, sector norte del Parque Nacional Pico Bonito.

Foto de James Adams en 2014.

fue captado cuando este individuo había ocupado como propia una plataforma para observadores de aves, instalada a $15 \mathrm{~m}$ de altura dentro del bosque. El registro de Bertín Lazo, en Protección (Santa Bárbara), fue el de un macho que estaba dentro del hueco de un tronco al momento de podar árboles para el mantenimiento de una servidumbre eléctrica.

\section{Discusión}

Los pocos registros de este marsupial en Honduras hasta la realización de este estudio, ha hecho suponer a las autoridades y técnicos hondureños, que esta especie pudiese estar en peligro de extinción. Sin embargo, con este estudio se aprecia que C. derbianus está bien distribuido en el país, incluyendo los amplios sectores de bosque fragmentado, cercanías a los parques nacionales y sectores caribeños semiurbanos.

El nuevo mapa de distribución de $C$. derbianus en Honduras presenta 40 sitios de encuentros, tanto históricos como nuevos, dejando ver con ello, la amplitud de su distribución en el país, ampliándose al norte, este y oeste (Figura 5). Sin embargo, no ha habido registros para la parte sur de Honduras. Esta región es parte de la franja de bosque muy seco tropical de Centroamérica; es posible que pueda encontrarse al norte-este del departamento de Choluteca (La Botija y La Caguasca), donde existen fragmentos de bosques latifoliados. Se efectuó un sondeo de 20 noches en estos dos bosques sin éxito.En este estudio se añade a la distribución nacional de esta especie, los departamentos de Copán,
El Paraíso y Yoro, como nuevos departamentos con presencia de $C$. derbianus, ampliando su área de distribución tanto en Oriente como en el Occidente del país. Marineros y Martínez (1998) mencionaron el encuentro de un animal cautivo en una feria colegial en Santa Rosa de Copán; con el presente estudio se confirma la presencia de esta zarigüeya en el departamento de Copán, a través de tres encuentros en el medio silvestre. De igual manera, se confirma el encuentro en el medio silvestre en el departamento de Comayagua en el sector sur de la represa El Cajón (R. Downing com. pers.), porque el registro histórico de esta zarigüeya en este departamento era de ubicación dudosa. Finalmente se añade el departamento de Yoro como nuevo registro departamental. La evidencia proviene del señor Rómulo Gutiérrez, quien fotografió un ejemplar al estar realizando un reconocimiento forestal en el caserío de Loma Larga, un área que drena sus aguas hacia el río Texiguat, y este al río Lean.

Con excepción de los registros en el río Santiago, Lancetilla y Texiguat, los demás registros presentados acá, corresponden a zonas muy alteradas, bosques secundarios, fincas, bosques riparios, cafetales, cacaotales e incluso zonas suburbanas, abarcando ecosistemas secos hasta muy húmedos. Los registros de Copán, Cortés, El Paraíso y todos los sitios del departamento de Santa Bárbara son sectores dominados por agropaisajes. Los departamentos de Atlántida y Santa Bárbara, tuvieron una mayor cantidad de reportes en comparación con otros departamentos, esto no tiene que ver con la existencia 


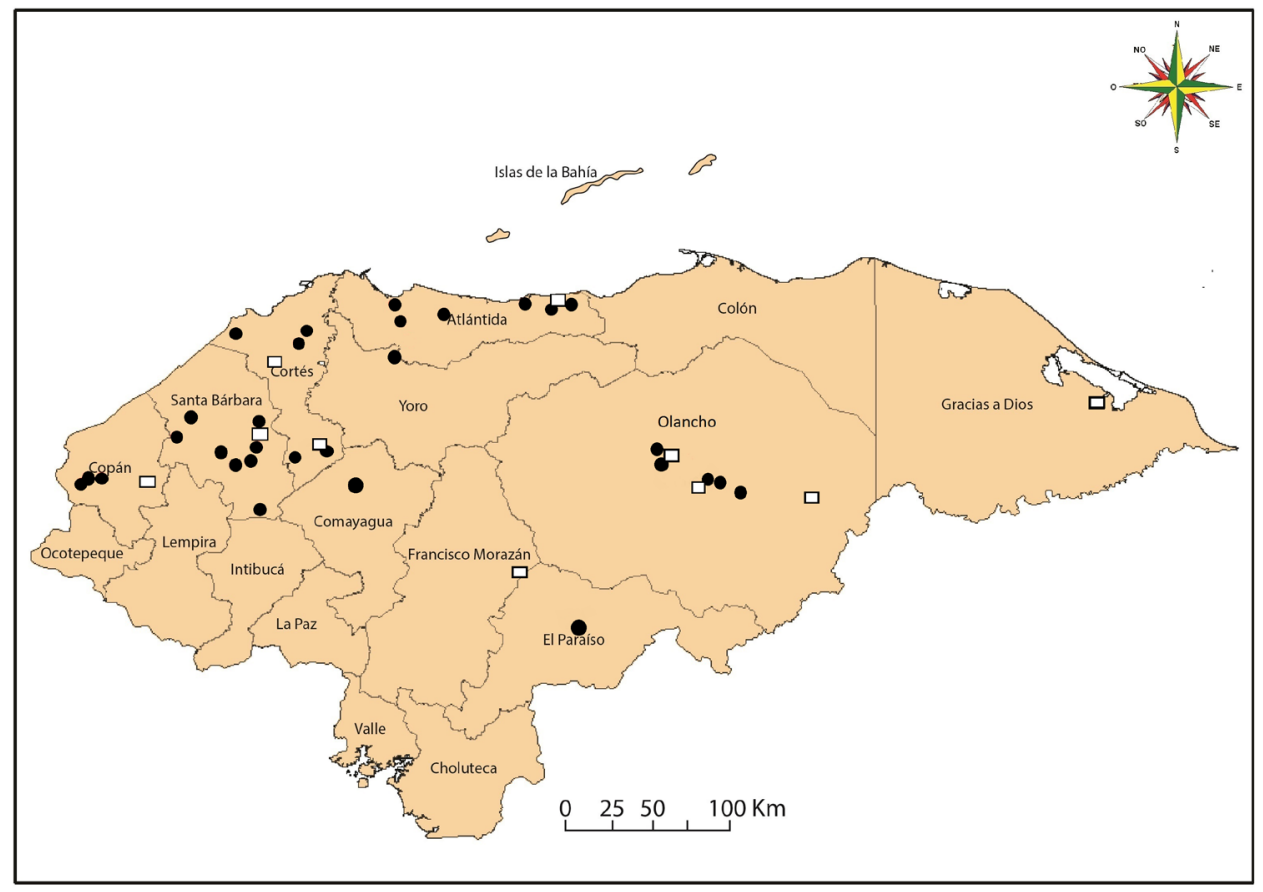

Figura 5. Mapa con los registros históricos (cuadrados) y los nuevos sitios (círculos) mencionado en este estudio.

de mayores poblaciones de este marsupial en esos sitios, sino a la presencia oportuna de técnicos y naturalistas que estuvieron trabajando o visitando esos sectores con mayor frecuencia.

Es probable que las poblaciones en algunas áreas protegidas, estén en buenas condiciones como en las áreas naturales de Los Naranjos, Cerro Santa Bárbara, PANACAM, Lancetilla, Punta Sal, Punta Izopo, Texiguat, Nombre de Dios y Pico Bonito, así como todas las áreas protegidas de los departamentos de Gracias a Dios y Colón, mas no así, en los sitios de agropaisajes.

El nombre "guazalillo dorado", dado por Gamero-Idiáquez en su libro sobre los mamíferos de Honduras de 1978, no es usado en ninguna parte del país, tampoco el nombre "zorra lanosa" que es una traducción del nombre en inglés. Por lo tanto, se debe considerar a este marsupial como un animal poco conocido por los pobladores rurales de Honduras y sin un nombre vernáculo generalizado.

\section{Conclusiones}

Caluromys derbianus, posee una distribución amplia en Honduras y su presencia con frecuencia en agropaisajes, hace suponer que las poblaciones en las áreas protegidas del país se encuentren en mejores condiciones. La especie no tiene presión de cacería por carecer de valor alimenticio, no encontramos evidencia de caza para consumo en las comunidades rurales o indígenas, tampoco tiene valor comercial para los grupos humanos porque rara vez se le ve en venta en las principales carreteras del país. Su única presión es la deforestación y la caza precautoria que los campesinos practican sobre algunos animales de monte para evitar posibles ataques a las aves de corral; este último argumento no ha sido confirmado aunque en las entrevistas algunas personas tenían la percepción que son peligrosos para estas aves.

Es posible que muchas de estas zarigüeyas sean cazadas para evitar daños en frutales de las huertas. Se le ha encontrado comiendo frutos de Piper sp. y frutos maduros de mangos (Josué Ramos G. común. pers.) en sectores cercanos a La Ceiba, Atlántida. En las visitas a las casas de los campesinos entrevistados, solamente en una ocasión se encontró una piel de $C$. derbianus adornando la pared de una casa, en la zona de amortiguamiento de la Reserva Biológica El Chile (Francisco Morazán) y otra piel en Agalta, al norte de Catacamas, Olancho. En otra ocasión, un animal fue encontrado cautivo en una finca en Santa Rosa de Copán, demostrando con ello que no es muy 
llamativo como animal de compañía o para adorno de exhibición.

Caluromys derbianus es catalogado como raro y de importancia para ecoturismo por la Resolución GG-APVS- 003-98 de la ya desaparecida AFE-COHDEFOR (1998), en donde la declaró como una especie silvestre en peligro de extinción o amenazada, por lo que aparece en el listado denominado "Lista de Especies de Animales Silvestre de Preocupación Especial para Honduras". La lista Roja de la UICN la consideró como "Vulnerable" en 1996; desde el año 2008 se la considera de preocupación menor (LC). Para la UICN las poblaciones están en decrecimiento, sin embargo, sigue siendo frecuente en todo su rango de distribución (Lew et al. 2008).

\section{Agradecimientos}

A los aportantes, colaboradores y revisores de este estudio: Olvin Calix to Ordoñez, Saby Cruz, Virginia Maklim Salinas, Bayardo Alemán, Danny Ordoñez, Josué Ramos Galdámez, Joel Mejía, Rómulo Gutiérrez, Ciro V. Navarro, Wendy Cerrato, Banny Quezada, Isaid Girón, Carlos Lazo Isaula, Bertín Lazo, Roberto Downing, Mario Espinal, Carlos O'Reilly, Pablo Bedrossian, Fausto Elvir, Jonathan Hernández, Bayardo Alemán, Jesse Kolar y Héctor Portillo. Se agradece, de igual manera, a los revisores de la revista por sus acertados comentarios.

\section{Literatura citada}

AFE-COHDEFOR, 1998. Departamento de Áreas Protegidas y Vida Silvestre. Resolución GG-APVS-003-98. Tegucigalpa: AFE-COHDEFOR.

Bianchi RA, Rosa A, Gatti RA, Méndes S. 2011. Diet of margay, Leopardus wiedii, and jaguarundi, Puma yagouaroundi, (Carnivora: Felidae) in Atlantic rainforest, Brazil. Zoologia. 28 (1): 127-32.

Bucher JE, Hoffman RS. 1980. Caluromys derbianus. Mammalian Species. 140: 1-4.
Collins LR. 1973. Monotremes and marsupials, a reference for zoological institutions. Smithson Inst Publ. 4888: $1-323$.

Eisemberg JF.1989. Mammals of the Neotropics: the northern Neotropics. Panamá, Colombia, Venezuela, Suriname, French Guiana. Vol 1. Chicago: The University of Chicago Press.

Fonseca R, Astúa D. 2015. Geographic variation in Caluromys derbianus and Caluromys lanatus (Didelphimorphia: Didelphidae). Zoologia (Curitiba). [acceso 4 de febrero de 2015] 32 (2): 109-22. Disponible en: http://dx.doi. org/10.1590/S1984-46702015000200002

Gamero-Ideáquez I. 1978. Mamíferos de mi tierra. Tegucigalpa: Banco Central de Honduras; 427 pp.

Gardner AL. 2008. Mammals of South America. Chicago: The University of Chicago Press.

Gómez de Silva H, Pérez-Villafaña M, Santos-Moreno JA. 1997. Diet of the spectacled owl (Pulsatrix perspicillata) during the rainy season in Northern Oaxaca, Mexico. $J$ Raptor Res. 31: 387-9.

Goodwin GG. 1942. Mammals of Honduras. Bull Am Mus Nat Hist. LXXIX: 107-95.

Hall ER, Kelson KR. 1959. The mammals of North America. Vol. 1, 2. New York: The Ronald Press Co.

Lew D, Soriano P, Cuarón AD, Emmons L, Reid F, Helgen K. [on line] 2008. Caluromys derbianus. The IUCN Red List of Threatened Species. Version 2014.2. [acceso 26 de septiembre de 2014]. Disponible en: www.iucnredlist.org

Marineros L, Martínez-Gallegos F. 1998. Guía de campo de los mamiferos silvestres de Honduras. Tegucigalpa: Instituto Nacional de Ambiente y Desarrollo.

Moreno R, Kays R, Samudio RJr. 2006. Competitive release in diets of ocelots (Leopardus pardalis) and puma (Puma concolor) after jaguar (Panthera onca) decline. J Mammal. 87 (4): 808-16.

Philips CJ, Jones JK. 1968. Additional comments on reproduction in the wolly opossum (Caluromys derbianus) in Nicaragua. J Mamm. 49: 320-1.

Reid FA. 2009. A field guide to the mammals of Central America and Southeast Mexico. $2^{\text {nd }}$ ed. Oxford: Oxford University Press.

UICN Red List of Threatened Species. [On line]. 2015 [acceso 26 de septiembre de 2014]. http://www.iucnredlist.org/ details $/ 3650 / 0$

Voss R, Jansa S. 2009. Phylogenetic relationships and classification of didelphid marsupials, an extant radiation of New World metatherian mammals. Bull Am Mus Nat Hist. 322: 1-177. doi: $10.1206 / 322.1$ 